\title{
Visual and quantitative evaluation of selected image combination schemes in ultrasound spatial compound scanning
}

Wilhjelm, Jens E.; Jensen, M.S.; Jespersen, S.K.; Sahl, B.; Falk, E.

Published in:

I E E E Transactions on Medical Imaging

Link to article, DOI:

10.1109/TMI.2003.822824

Publication date:

2004

Document Version

Publisher's PDF, also known as Version of record

Link back to DTU Orbit

Citation (APA):

Wilhjelm, J. E., Jensen, M. S., Jespersen, S. K., Sahl, B., \& Falk, E. (2004). Visual and quantitative evaluation of selected image combination schemes in ultrasound spatial compound scanning. I E E E Transactions on Medical Imaging, 23(2), 181-190. https://doi.org/10.1109/TMI.2003.822824

\section{General rights}

Copyright and moral rights for the publications made accessible in the public portal are retained by the authors and/or other copyright owners and it is a condition of accessing publications that users recognise and abide by the legal requirements associated with these rights.

- Users may download and print one copy of any publication from the public portal for the purpose of private study or research.

- You may not further distribute the material or use it for any profit-making activity or commercial gain

- You may freely distribute the URL identifying the publication in the public portal 


\title{
Visual and Quantitative Evaluation of Selected Image Combination Schemes in Ultrasound Spatial Compound Scanning
}

\author{
J. E. Wilhjelm*, M. S. Jensen, S. K. Jespersen, B. Sahl, and E. Falk
}

\begin{abstract}
Multi-angle spatial compound images are normally generated by averaging the recorded single-angle images (SAIs). To exploit possible advantages associated with alternative combination schemes, this paper investigates both the effect of number of angles $\left(N_{\theta}\right)$ as well as operator (mean, median, mean-excluding-maximum (mem), root-mean-square (rms), geometric mean and maximum) on image quality (tissue delineation and artifacts), speckle signal-to-noise ratio $\left(\mathrm{SNR}_{s}\right)$ and contrast. The evaluation is based on in vitro $\mathrm{SAI}\left( \pm 21^{\circ}\right.$ in steps of $\left.\Delta \theta=7^{\circ}\right)$ of formalin fixed porcine tissue containing adipose, connective and muscular tissue. Image quality increased with number of angles up to $\pm 14^{\circ}$ after which the improvements became debatable. The mem and median operators, which try to render the images more quantitatively correct by suppressing strong echoes from specular reflectors, provide some improvement in this regard. When combining the SAI with the mean operator, the $\mathrm{SNR}_{s}$ increases-in general一with $N_{\theta}$. For $N_{\theta}=2$, the $\mathrm{SNR}_{s}$ increases with $\Delta \theta$ as expected. When $N_{\theta}=7$, the highest $\mathrm{SNR}_{s}$ is obtained for the mem, rms, and geometric mean operators, while the lowest $\mathbf{S N R}_{s}$ is obtained for the maximum operator. When comparing $\mathbf{S N R}_{s}$ for adipose and fibrous tissue, the level is close to 1.91 for adipose tissue but only 1.7 for fibrous tissue which contain relatively few organized scattering structures.
\end{abstract}

Index Terms-Anatomy, compound operators, contrast, histology, spatial compound ultrasound, speckle signal-to-noise ratio.

\section{INTRODUCTION}

$\mathbf{M}$ ULTI-ANGLE COMPOUND IMAGING (MACI) offers noticeable advantages in image improvements, due to the reduced angle-dependence and the reduced speckle in the compound image compared with conventional B-mode imaging. This has been demonstrated both in vitro [8], [12] and in vivo [1], [3], [5], [11], [4]. In this technique, images are recorded from a number of different angles (typically 3 to 9 ), and these single-angle images (SAIs) are then combined to form the compound image. Compound images can be

Manuscript received September 25, 2002; revised September 26, 2003. The Associate Editor responsible for coordinating the review of this paper and recommending its publication was M. Insana. Asterisk indicates corresponding author.

*J. E. Wilhjelm is with the Center for Arteriosclerosis Detection with Ultrasound (CADUS), Ørsted-DTU, Technical University of Denmark, Bldg. 348, DK-2800 Kgs. Lyngby, Denmark (e-mail: jw @ oersted.dtu.dk; Homepage: www.oersted.dtu.dk/ jw/cadus).

M. S. Jensen is with the Center for Arteriosclerosis Detection with Ultrasound (CADUS), Ørsted.DTU, Technical University of Denmark.

S. K. Jespersen is with Novo Nordisk A/S, Brennum Park, DK-3400 Hillerød, Denmark.

B. Sahl and E. Falk are with the Institute of Experimental Clinical Research, Aarhus University Hospital (Skejby), DK-8200 Aarhus N, Denmark.

Digital Object Identifier 10.1109/TMI.2003.822824 recorded with a fine pitch linear array transducer connected to an ultrasound system with a sufficiently high number of parallel channels, allowing for beam-steering with sufficiently low disturbance from grating lobes.

In most investigations of this technique so far, the individual SAIs have been simply averaged to form the compound image. In order to study in more details different alternative ways of combining the images, this paper reports on various combination schemes based on SAIs recorded from porcine tissue. By embedding the tissue in an agar block that contained a series of fiducial markers, the tissue could — after scanning—be sliced and photographed at planes that exactly matched the ultrasound scan planes [7]. The macroscopic images hereby recorded were then used as a geometrical reference, when evaluating the various imaging schemes. The tissue slices were finally analyzed histologically, so that the different materials in the macroscopic image could be identified.

This study used a transducer geometry (array length of $40 \mathrm{~mm}$ ) and center frequency, 7.5 MHz, specifically optimized for scanning of the carotid artery.

\section{MATERIALS AND METHODS}

\section{A. Biological Materials}

An approximately 40-mm-long piece of porcine fatty muscle was formalin fixed while folded along an 8-mm metal rod, to have the fixed tissue somewhat mimic part of a (sclerotic) vessel wall. With this kind of porcine tissue, the differences between the tissue types are typically larger than for a sclerotic vessel and the borders between the different tissues are more well defined. After 24 hours of fixation, the tissue was placed-supported by two wires - in a molding frame and degassed liquid agar ( $1 \%_{\text {weight }}$ agar-agar) was slowly poured into the frame. When filled, a lid with rows of rectangular openings was placed on top of the liquid agar. The agar block then hardened. When set, the agar block contained two rows of fiducial markers on the top that could be recognized both by ultrasound and later by the operator, who sliced the agar block.

\section{B. Experimental Scanning System}

The experimental system is illustrated in Fig. 1. It consists of: 1) a MACI (off-line) ultrasound scanner (denoted the Xtra system) developed at our laboratory and previously described, [8]; 2) a 192-element 7.5-MHz linear array transducer $40 \mathrm{~mm}$ wide [8] ( $\lambda=0.2 \mathrm{~mm}$ at $\left.\left.20^{\circ} \mathrm{C}\right) ; 3\right)$ a tank with degassed demineralized water at $\sim 20^{\circ} \mathrm{C}$ into which the agar block was sub- 


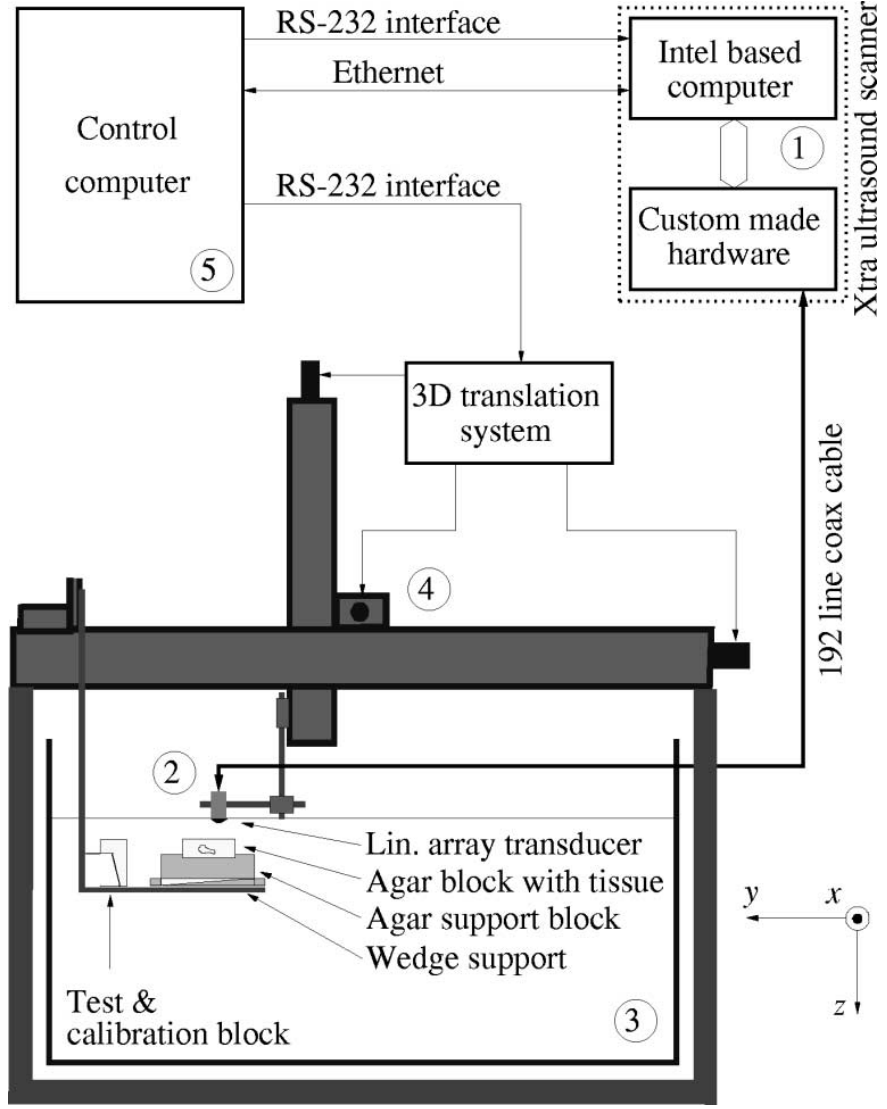

Fig. 1. Illustration of the entire measurement system. The linear array transducer is recording images in the $x-z$ plane and is moved mechanically along the $y$-direction. The test block is used for functionality test of the ultrasound system.

merged; 4) a three-dimensional (3-D) translation system capable of translating the transducer; and 5) a control computer running Matlab (Mathworks Inc., Natic, MA).

The control computer was connected via an RS232 interface to the 3-D translation system so that the transducer could be translated in a number of parallel scan planes automatically. The control computer was also connected via another RS232 interface to the Xtra system, which under normal operation was running MS-DOS. In this way, the Xtra system could remotely be made to record a set of SAIs and store these to the local disk. After an entire scanning session, the control computer then, also via the RS232 interface, forced the Xtra system into MS-Windows mode, so that an additional, much faster interface (Ethernet) could be used for transfer of the data from the Xtra system to the control computer. Data was saved on disk as raw radio frequency (RF) beamformed signals (the original single-element signals were digitized into 12 bits).

In this in vitro study in water, time-gain compensation (TGC) was later applied to each individually received signal as a $20-\mathrm{dB}$ gain per $\mathrm{cm}$ "screen" depth in tissue regions and $0-\mathrm{dB} / \mathrm{cm}$ gain in agar and water. The former value was found as the TGC value, where the mean gray scale level of the least anisotropic tissue type was the most constant (i.e., the 35 adipose tissue regions, as will be explained in Section II-F). The value is a little higher than the commonly accepted average attenuation in vivo which is $1 \mathrm{~dB} / \mathrm{MHz} / \mathrm{cm}$ travelled.

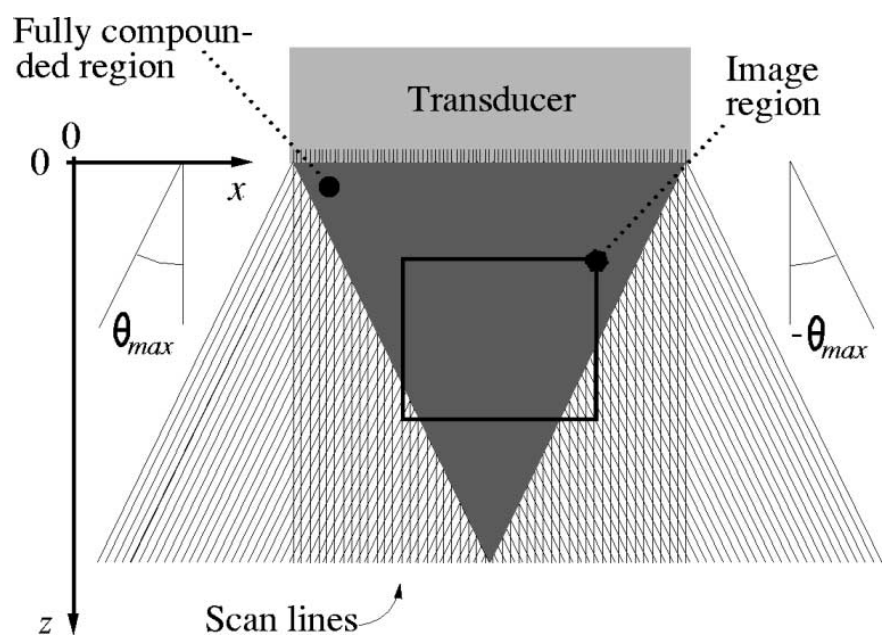

Fig. 2. Illustration showing the location of the image region (inside the FCR) that is used in this study. The region is located slightly to the right of the FCR. Drawing not to scale.

\section{Ultrasound Scanning Procedure}

The agar block with the porcine tissue was placed in the scanning tank and 65 cross-sectional images inter-spaced $0.5 \mathrm{~mm}$ were recorded by using the angles: $\theta \in\left[-21^{\circ},-14^{\circ},-7^{\circ}, 0^{\circ}, 7^{\circ}, 14^{\circ}, 21^{\circ}\right]$, where the angular separation, $\Delta \theta=7^{\circ}$. The envelope of the recorded (beamformed) $\mathrm{RF}$ data was scan converted to form raw SAIs, $I(z, x, \theta ; y)$ associated with angle $\theta$ and cross-sectional scan plane $y$ [8]. $z$ and $x$ constitute the image plane. Each image consists of 192 parallel scan lines. As previously described [8], the fully compounded region (FCR) will be a triangle, and in this study, the majority of the tissue specimen was inside this triangular region. The images presented in this paper are extracted as a rectangular region mostly inside the FCR, as shown in Fig. 2. The $z$ and $x$ axes on the ultrasound images correspond to the coordinate system in Fig. 2.

\section{Slicing of Tissue}

After ultrasound scanning, the agar block was cooled to $5{ }^{\circ} \mathrm{C}$ and then sliced at the fiducial marker locations, with an interslice distance of $2.5 \mathrm{~mm}$. After each "cut" with the knife, a photograph was recorded of the 2.5 - $\mathrm{mm}$ tissue slice and this photograph was later calibrated to obtain a metric correct image. The sliced tissue was analyzed histologically. More details can be found in [7].

\section{E. Compound Image Formation}

All the images considered here are based on the raw envelope detected and scan converted data, unless specifically noted otherwise. When displayed in decibels, the image underwent the transform $20 \log _{10}(\cdot)$, which is a more discernible approach, than the logarithmitizing and image adjustments that are normally done in most ultrasound scanners.

The results from two typical scan planes out of the 65 crosssectional scan planes are considered in the qualitative part of this paper. These two scan planes are denoted $y_{1}$ and $y_{2}$ and anatomical photographs exist for both of these scan planes. To obtain an easily readable decibel scale (with a maximum of $0 \mathrm{~dB}$ ), the 
14 SAIs recorded from these two scan planes were first normalized — with the same constant—so that the maximal pixel value corresponded to unity

$$
I_{N}(z, x, \theta ; y)=\frac{I(z, x, \theta ; y)}{\max _{z, x, \theta, y_{1}, y_{2}}\{I(z, x, \theta ; y)\}} .
$$

The mean image (the conventional compound image) can be constructed by averaging the SAIs over the angles desired

$$
I_{\text {mean }}(z, x ; y)=\operatorname{mean}_{\theta}\left\{I_{N}(z, x, \theta ; y)\right\} \text {. }
$$

In the averaging technique, echoes from specular reflectors can dominate the final image. This angle-dependent effect is often an advantage when viewing and subjectively interpreting the image, but if quantitative information should be derived from the image, such strong echoes are undesired. A possible way to reduce the influence of specular reflectors-that is also used in statistics as an estimate of the average, when the number of samples is small-is to use the median instead of the mean

$$
I_{\text {med }}(z, x ; y)=\underset{\theta}{\operatorname{median}}\left\{I_{N}(z, x, \theta ; y)\right\} \text {. }
$$

A quite similar approach can be implemented by modifying (2), so that, for each pixel, the average is taken over all angles, excluding the angle with the largest value. If the remaining (in this study, six) pixels are denoted $I_{N}^{\prime}(z, x, \theta ; y)$, then this approach ("mean excluding maximum") can be written as

$$
I_{\text {mem }}(z, x ; y)=\operatorname{mean}_{\theta}\left\{I_{N}^{\prime}(z, x, \theta ; y)\right\} .
$$

An approach that weights the individual values differently, depending on their value, can be realized by using the root-meansquare (rms) value

$$
I_{\mathrm{rms}}(z, x ; y)=\sqrt{\operatorname{mean}_{\theta}\left\{I_{N}(z, x, \theta ; y)^{2}\right\}}
$$

which, assuming that $I$ is Rayleigh distributed [2], is proportional to the maximum-likelihood estimate of the mean [9]. For completeness, strictly speaking, $I$ will only be Rayleigh distributed [13] in the case of fully developed speckle. Finally, from an implementation point of view, it is relevant to ask, what the result is in case the averaging process is done after the logarithmization

$$
L_{\log }(z, x ; y)=\frac{1}{N_{\theta}} \sum_{\theta} 20 \log _{10}\left(I_{N}(z, x, \theta ; y)\right)
$$

where $N_{\theta}$ is the number of angle used. Rearranging and removing $\log$ gives

$$
I_{\Pi}(z, x ; y)=\left(\prod_{\theta} I_{N}(z, x, \theta ; y)\right)^{1 / N_{\theta}}
$$

which is the geometric mean. Note that (7) does not include any truncation of the logarithmitized image, as could be the case in a commercial scanner. For completeness, early implementations [2] of compound techniques applied the maximum operator

$$
I_{\max }(z, x ; y)=\max _{\theta}\left\{I_{N}(z, x, \theta ; y)\right\} .
$$

\section{F. Quantitative Analysis}

This section deals with quantitative measures of specific aspects of the images generated by the operators in Section II-E. Specifically, the variation in the image due to speckle is measured in terms of the speckle signal-to-noise ratio (SNR) (also referred to as "signal-to-noise ratio at a point") while the ability

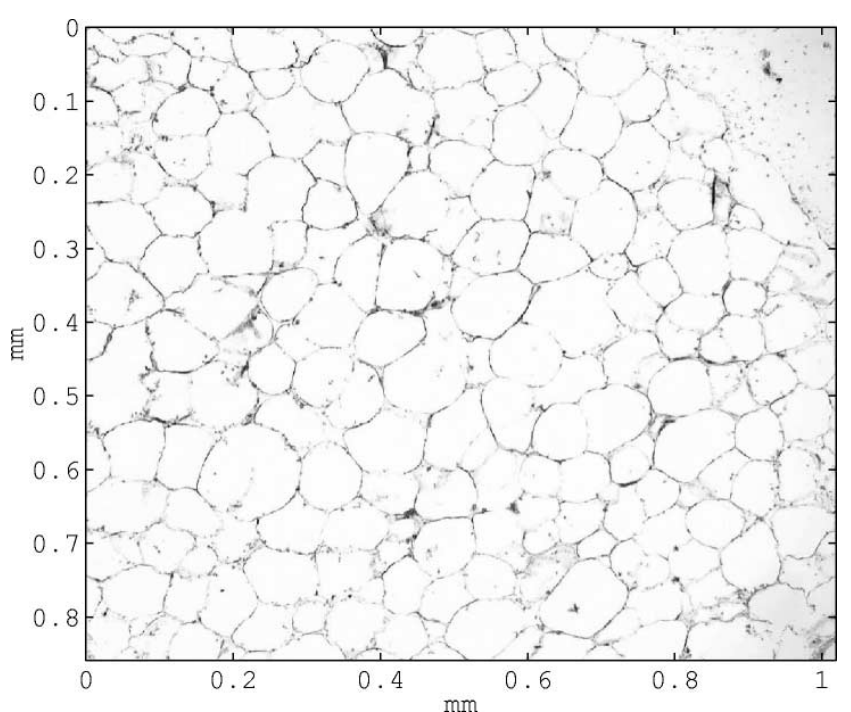

(a)

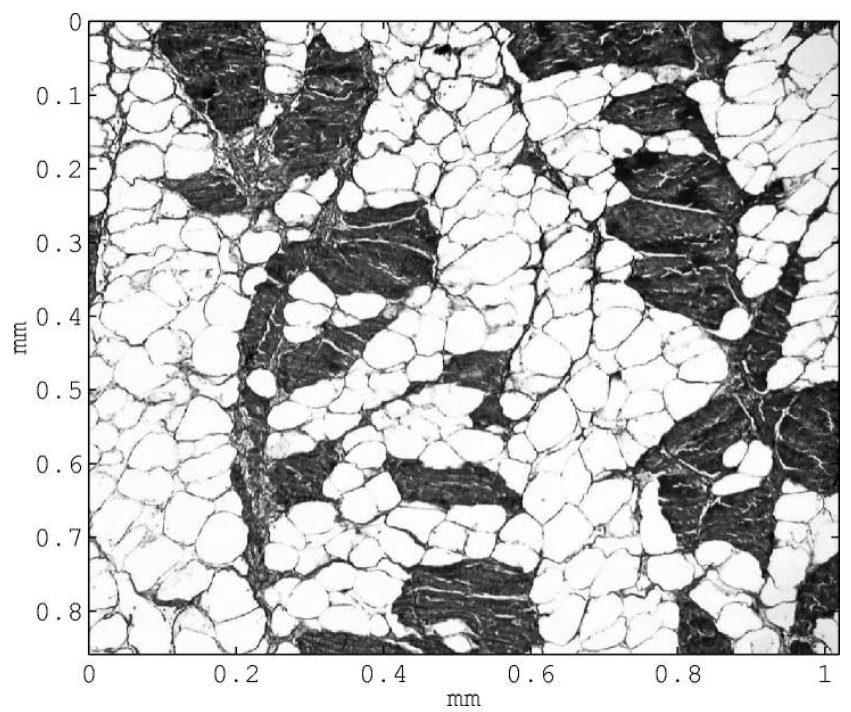

(b)

Fig. 3. Example of elastin trichrome stained images of (a) adipose tissue and a mixture of adipose and fibrous tissue (appearing blue). The area of each photograph corresponds to approximately $22 \lambda^{2}$.

to distinguish between tissues of different types is investigated by calculation of contrast between these.

The above measures were calculated for regions of different tissue. Maps showing the tissue types present were found this way: The histological images indicate four types of tissues: tissue with a high content of muscle fibers, tissue with a high content of lipid, tissue with a high content of connective tissues and finally small vessels. These four tissue types were identified and outlined on templates for the 12 image planes that were represented by an anatomical image, as described in detail in [7]. This resulted in 12 different maps that for each pixel in the ultrasound image indicated the tissue type. From the group of four tissue types, the two least anisotropic tissues were selected as follows: regions with a high lipid content (adipose tissue) and regions with a high content of fibrous tissue, where the fibers were randomly oriented [the latter did not contain sheets 


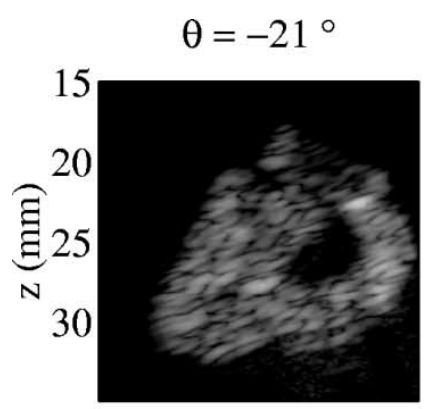

$$
\theta=-14^{\circ}
$$
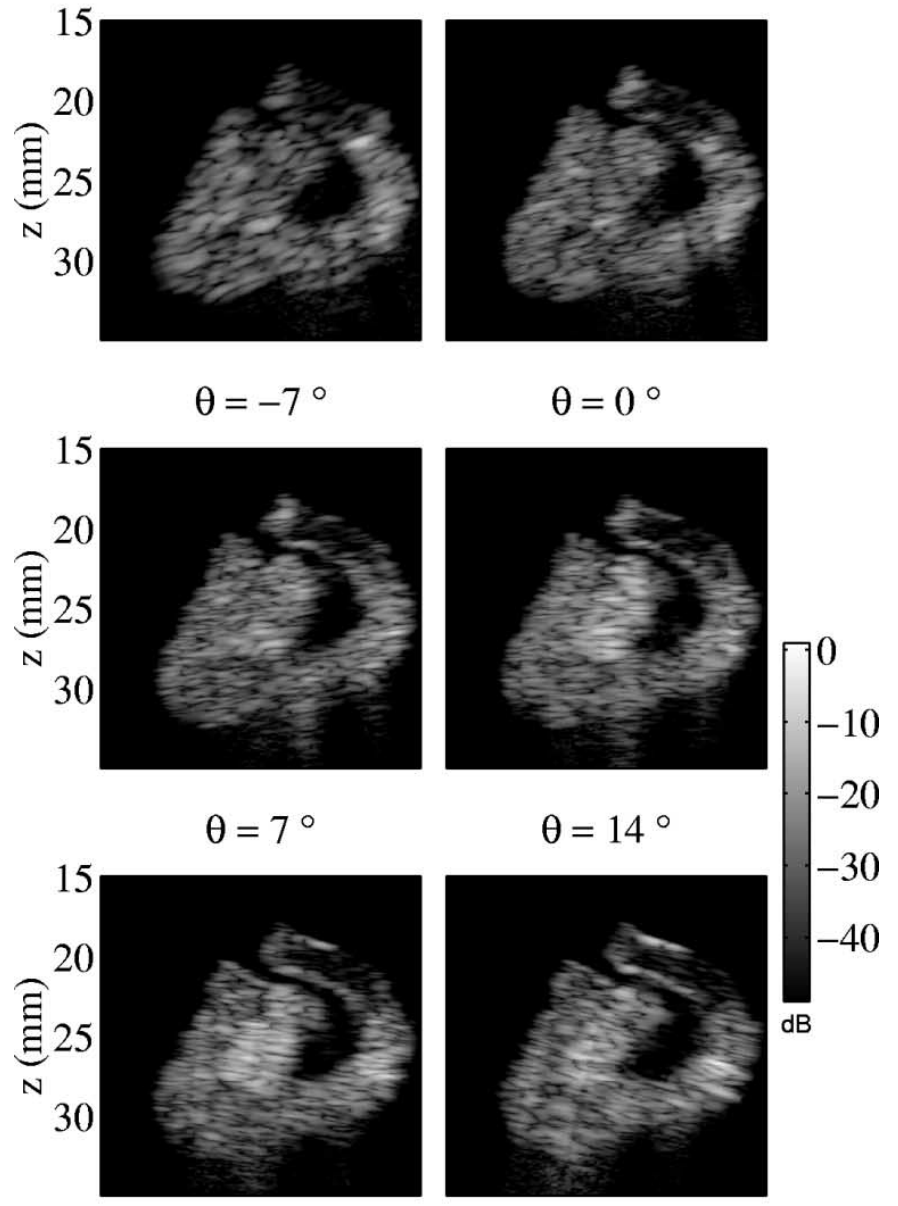

$\theta=21^{\circ}$

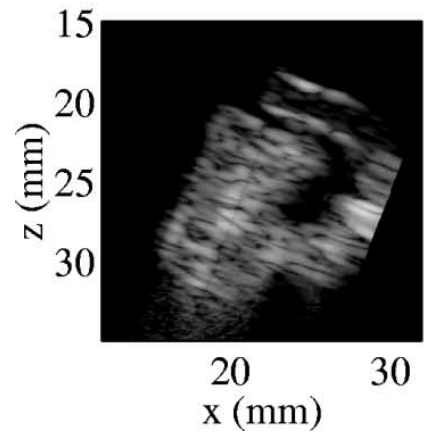

Fig. 4. The normalized seven SAIs for scan plane $y_{1}$. Occasional "weak lines" in these images is due to the TGC compensation (e.g., as seen in the $21^{\circ}$ image).

of fibrous tissue (septa)]. Histological images of these two tissue types can be seen in Fig. 3 .

To partly suppress the influence from the echo between the tissue region and the bordering tissues, a narrow band along the border of the tissue region was identified and only the tissue region inside of this band was analyzed. The width of this border band, $\sim 0.3 \mathrm{~mm}$, corresponded approximately to the $-3 \mathrm{~dB}$ width of the point spread function (psf). Only tissue regions that hereafter were larger than $4.7 \lambda^{2}\left(0.2 \mathrm{~mm}^{2}\right)$ were analyzed; A total of 35 fibrous tissue regions and a little more than 35 adipose tissue regions fulfilled this criterion.
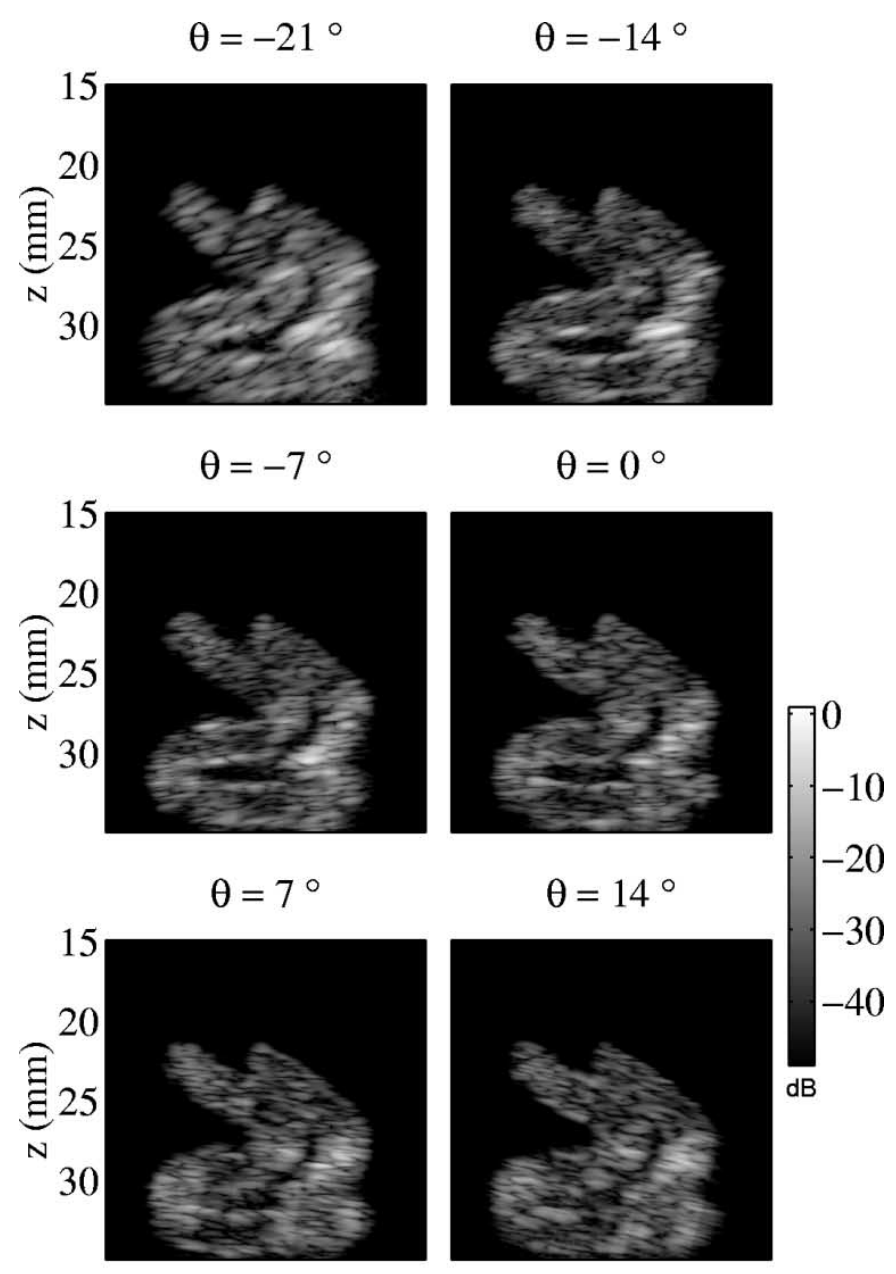

$\theta=21^{\circ}$

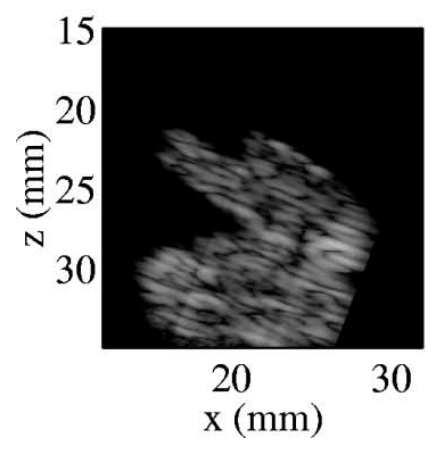

Fig. 5. The normalized seven SAIs for scan plane $y_{2}$.

Letting subscript $\bullet$ denote any of the seven image combination schemes, the mean and standard deviation of $I_{\bullet}(z, x ; y)$ inside the 35 adipose tissue (lip) regions and the 35 fibrous (fib) regions, were calculated and denoted: $\mu_{\text {lip }}^{(n)}, \sigma_{\text {lip }}^{(n)}, \mu_{\text {fib }}^{(n)}$, and $\sigma_{\text {fib }}^{(n)}$, where $n \in[1: 35]$ is the region number in the 3-D data set. All calculations were done on the nonlogarithmitized data.

The speckle SNR (inverse of speckle contrast) was calculated for both tissue types

$$
\operatorname{SNR}_{s, \text { lip }}^{(n)}=\frac{\mu_{\text {lip }}^{(n)}}{\sigma_{\text {lip }}^{(n)}} \quad \operatorname{SNR}_{s, \text { fib }}^{(n)}=\frac{\mu_{\text {fib }}^{(n)}}{\sigma_{\text {fib }}^{(n)}} .
$$



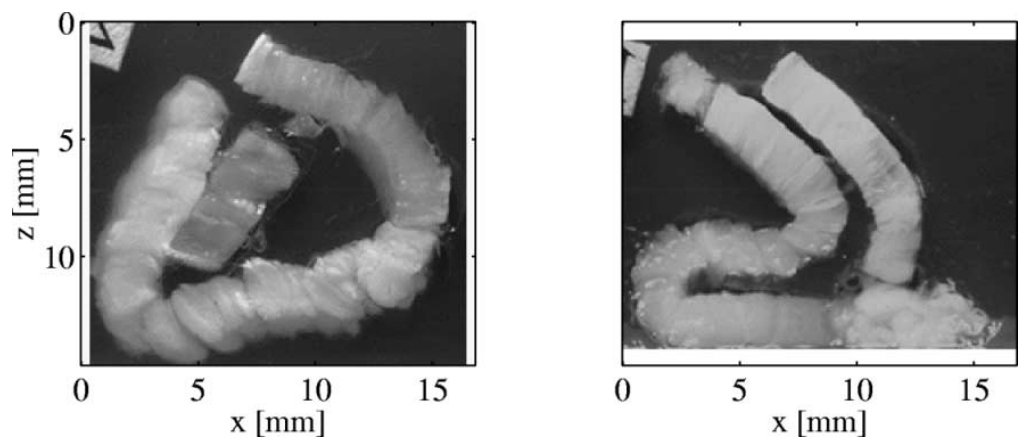

Fig. 6. Anatomical photographs of the porcine tissue corresponding to scan planes $y_{1}$ and $y_{2}$, respectively. Compared to the ultrasound images, the motives (tissue outlines) on these photographs have a slightly distorted geometry due to the cutting procedure [7].
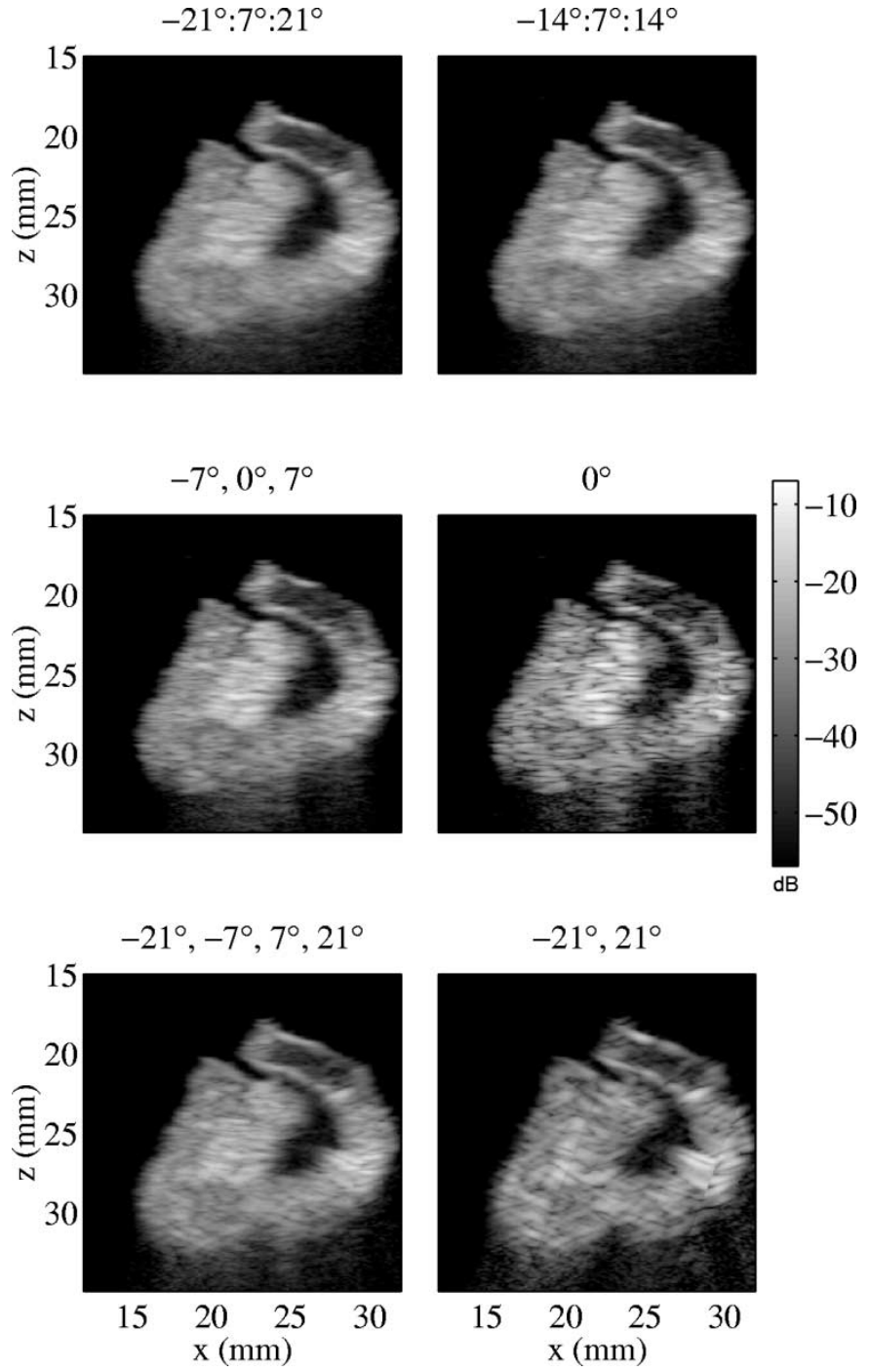

Fig. 7. The effect of reducing the number of SAIs used to generate the mean image for scan plane $y_{1}$. The actual angles used are written above each image.

The contrast between lipid rich and fibrous rich tissue was calculated as

$$
C^{(n)}=\frac{\left|\mu_{\text {lip }}^{(n)}-\mu_{\mathrm{fib}}^{(n)}\right|}{\sqrt{\left(\sigma_{\mathrm{lip}}^{(n)}\right)^{2}+\left(\sigma_{\mathrm{fib}}^{(n)}\right)^{2}}}
$$
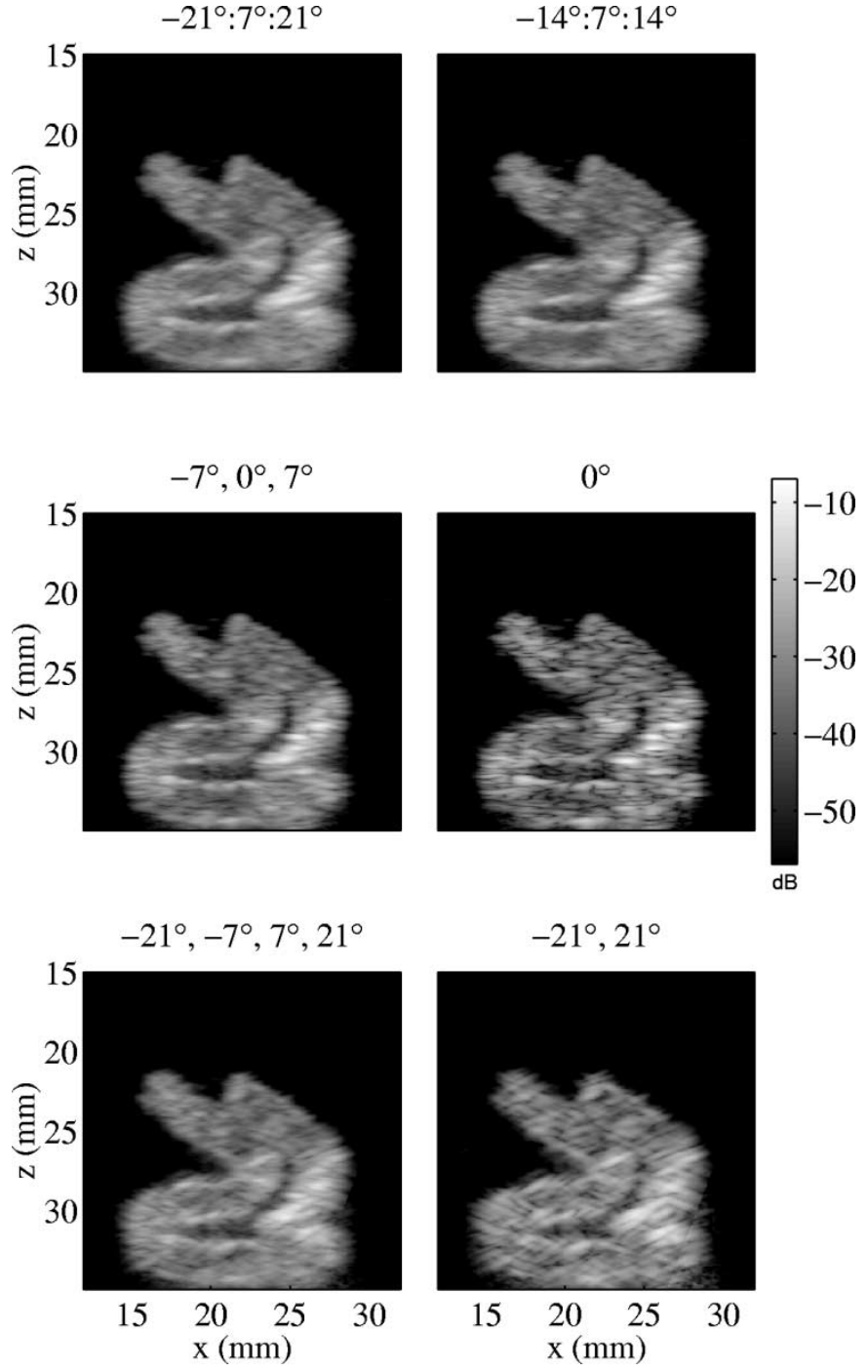

Fig. 8. The effect of reducing the number of SAIs used to generate the mean image for scan plane $y_{2}$. The actual angles used are written above each image.

which is an adapted version of the contrast-to-noise ratio in the sense that (10) here represents the "contrast-between-tissues" ratio. This contrast measures both the difference in amplitude value between the two tissues as well as the variation in amplitude inside the tissue regions. For the mean operator, it is observed, that when increasing the number of SAIs, the difference in mean value between two given regions does not change, but 

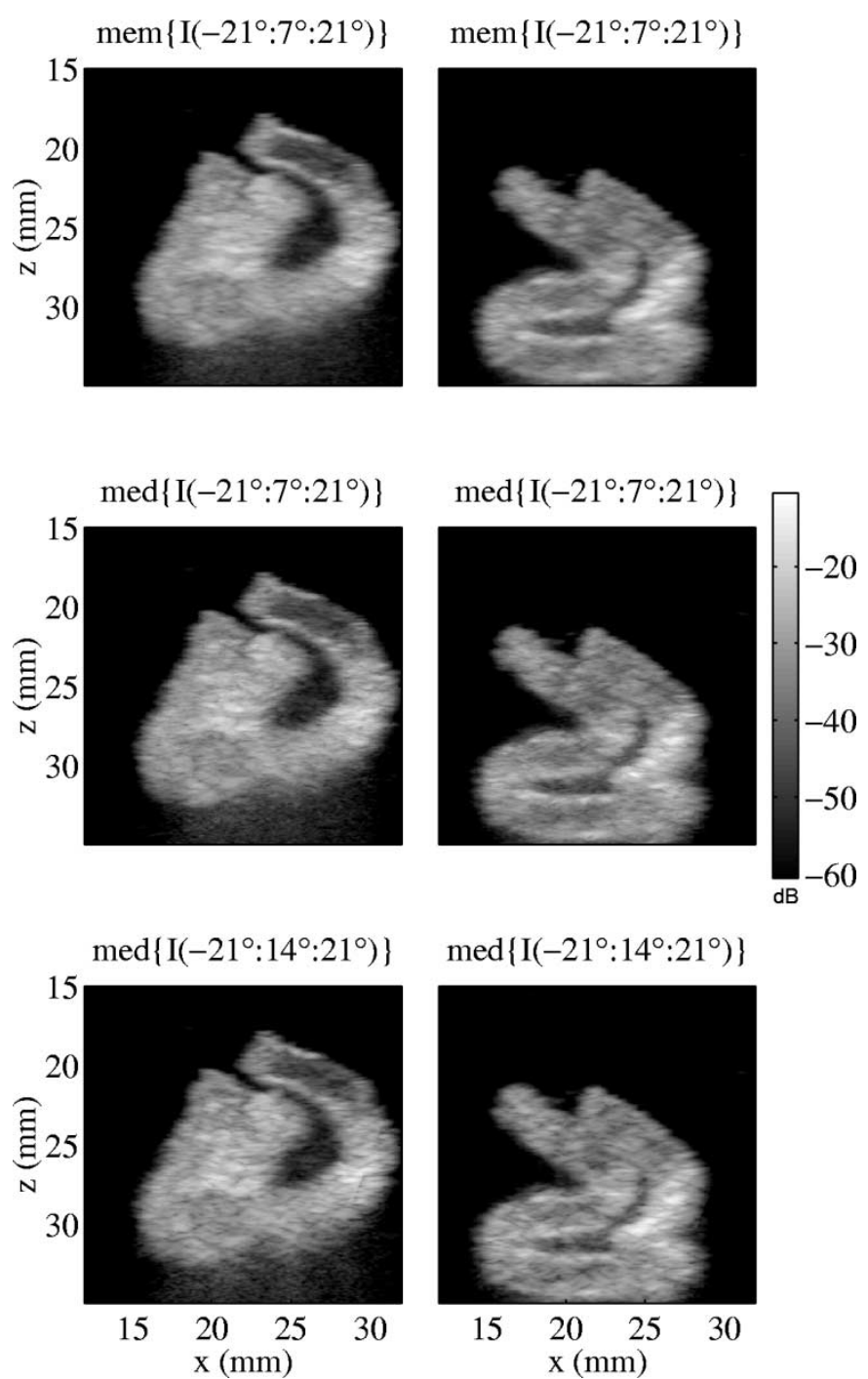

Fig. 9. First row: For each pixel, these images represent the mean over all angles, except the angle with the maximal value. Second row: The median image for each of the two scan planes using all angles. Third row: Same as the second row, except that only the angles $-21^{\circ},-7^{\circ}, 7^{\circ}$, and $21^{\circ}$ are used.

the variance for each region decreases thereby giving a higher contrast in (10).

\section{RESULTS}

\section{A. Image Visualization}

The normalized seven SAIs recorded from scan plane $y_{1}, I_{N}\left(z, x, \theta ; y_{1}\right)$ are shown in Fig. 4 while the images for scan plane $y_{2}$ are shown in Fig. 5. Note that the tissue was not completely inside the FCR, as seen from the $\theta=21^{\circ}$ image. The corresponding macroscopic photographs of scan plane $y_{1}$ and $y_{2}$ are shown in Fig. 6. Even though slightly geometrically distorted due to the cutting process involved with creating these images [7], the images in Fig. 6 reveal the delineation of the tissue scanned. Fig. 7 shows the mean image, $I_{\text {mean }}\left(z, x ; y_{1}\right)$, when the number of angles is gradually reduced by either reducing $\theta_{\max }$ or increasing $\Delta \theta$. Fig. 8 shows the same for scan plane $y_{2}$. The image denoted $0^{\circ}$ corresponds to the conventional B-mode image. Fig. 9 shows, for each scan plane, the "mean excluding maximum" image as well as two median images using different combinations of angles. Fig. 10 shows the rms images for both scan planes calculated according to (5). Fig. 11 shows the images for the two scan planes, when the logarithm is taken before the averaging, i.e., calculated according to (7). Finally, Fig. 12 shows the images for the two scan planes, when using the maximum operator.

Note that the notation $-21^{\circ}: 7^{\circ}: 21^{\circ}$ means the angles from $-21^{\circ}$ to $21^{\circ}$ in steps of $\Delta \theta=7^{\circ}$. The images in Figs. 4-11 are all plotted with the same decibel scale. On each individual figure, this scale spans exactly $50 \mathrm{~dB}$. Thus, each gray scale bar might be offset from each other. This way the gray scale is fully used (as would be the case on a commercial scanner), but the lower the values of the gray scale bar, the lower the signal value, and thus, the higher the potential influence from noise.

\section{B. Speckle Level and Contrast}

The $\mathrm{SNR}_{\mathrm{S}}$ and the contrast were evaluated for the 35 adipose and the 35 fibrous tissue regions, as explained in Section II-F. These measures are plotted for the different image operators for a range of angle combinations in Table I: The first eight lines show various combinations of $N$ and $\Delta \theta$ for the mean operator. The next seven lines show the results for each individual angle. The last five lines show the results for the remaining five operators, using all seven angles. The results are shown as the group of bares to the right.

The first column of bars shows the contrast while the following two columns of bars show $\mathrm{SNR}_{\mathrm{S}}$ for the fibrous and adipose tissue regions. These three sets of bars are calculated by averaging the 35 tissue regions. The associated standard deviation was maximally about $30 \%$ for the $\mathrm{SNR}_{\mathrm{S}}$ and $65 \%$ for the contrast.

The last column of these bars-with $\mathrm{SNR}_{S}$ - gives an estimate of the theoretical speckle SNR calculated from [10]

$$
\mathrm{SNR}_{s}=1.91 \sqrt{\frac{N}{1+2(N-1) \rho / N}}
$$

where $\rho$ is the correlation between two neighboring angles. With the system parameters for the Xtra scanner, $\rho=0.2$ at the focal point [8]. In (11), it is assumed that the correlation between scan angles separated further than $\Delta \theta$ is zero. Finally, in deriving (11), it was assumed that the echoes are the result of scattering from a high number of tiny scattering centers, which are (uniformly) randomly distributed in space [10]. This condition is not fully fulfilled for the adipose and fibrous regions used here and depicted in Fig. 3. $\mathrm{SNR}_{S}$ is only calculated for the mean operator and for the SAIs (in the latter case, $N=1$ and $\mathrm{SNR}_{S}=1.91$ ).

\section{DISCUSSION}

\section{A. Visual Interpretation}

The images in Section III-A can be compared in terms of: 1) the delineation of the tissue relative to the anatomical reference images in Fig. 6; 2) (obvious) image artifacts; and 3) degree of speckle as well as the overall pixel level relative to the background, as revealed by the decibel range of the gray scale bar. 

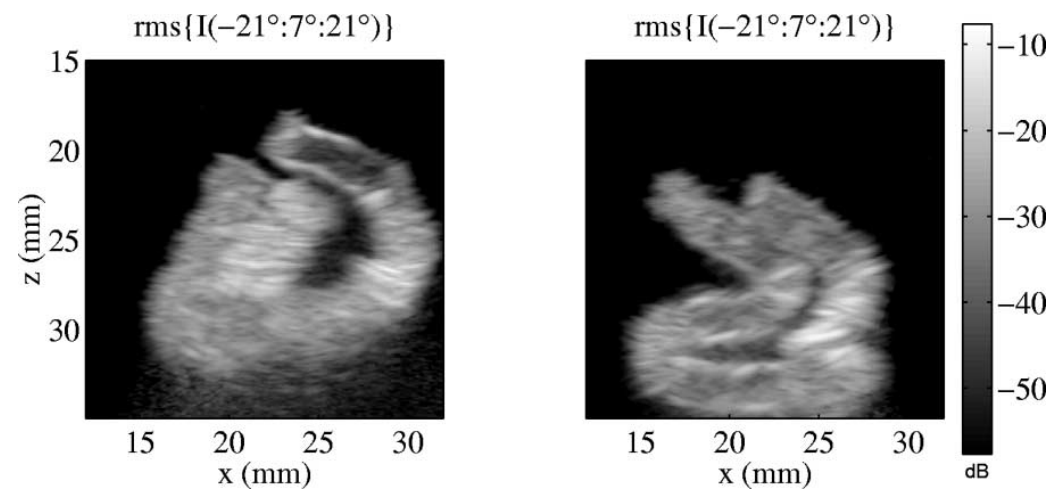

Fig. 10. The rms images for scan plane $y_{1}$ and $y_{2}$ using all angles.
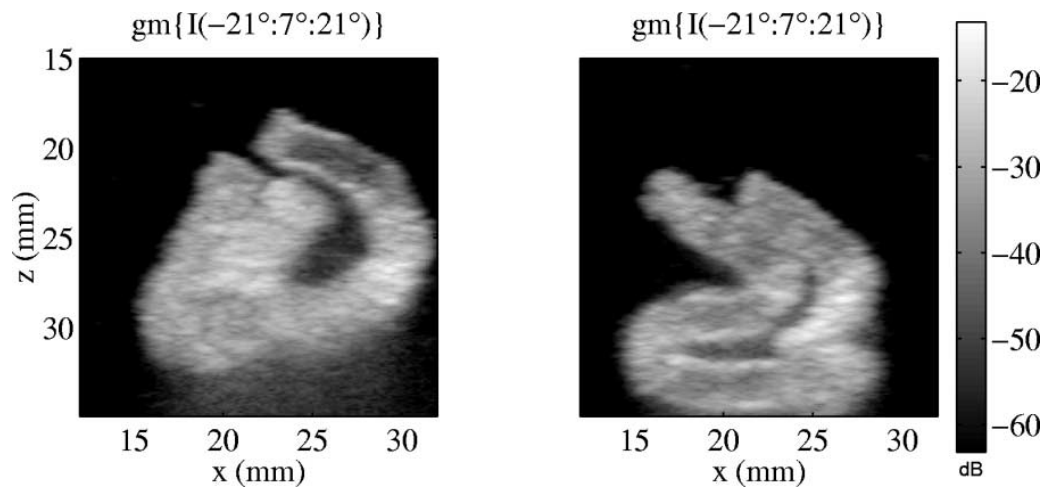

Fig. 11. Compound images for the two scan planes, when the logarithm is taken before averaging (i.e., the geometric mean in (7)), using all angles.
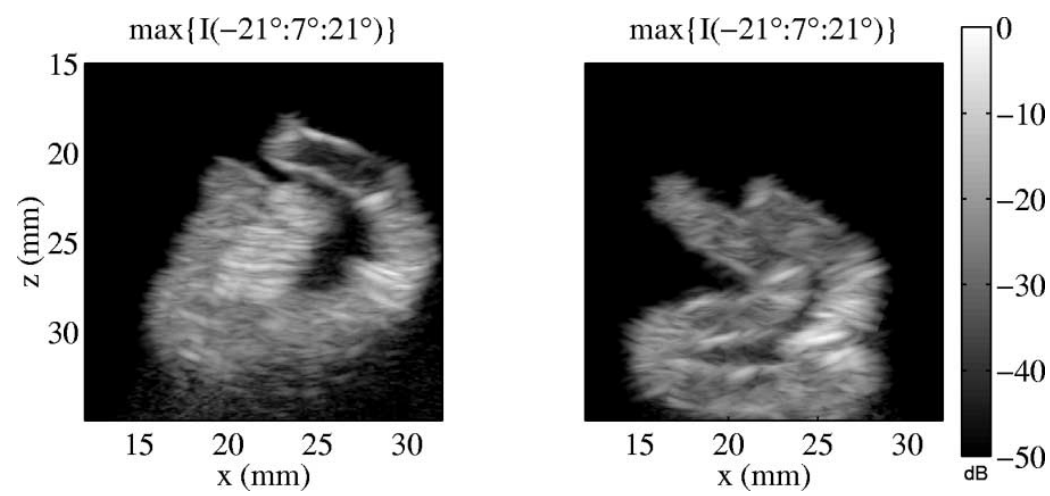

Fig. 12. Compound images for the two scan planes, when using the maximum operator.

From the SAIs in Figs. 4 and 5 the change in size and shape of the psf, as a function of scan angle, can be appreciated. These images also reveal the angle-dependences present in the images, due to specular reflectors such as interface between tissue and agar (e.g., the interface in Fig. 4 at 1 o'clock, from invisible at $-21^{\circ}$ to clearly visible at $21^{\circ}$ ), anisotropic tissue such as muscle (e.g., Fig. 4 at $x=20-24 \mathrm{~mm}, z=22-26 \mathrm{~mm}$ ) and occasionally strong constructive interference from closely spaced inhomogeneities (e.g., what could be a "speckle dot" in Fig. $4,-21^{\circ}$ at about 2 o'clock).

When considering the mean images in Figs. 7 and 8, it can be seen that removing the two most extreme scanning angles $\left( \pm 21^{\circ}\right)$, so that the number of angles is decreased from seven to five, only have a minor impact on the delineation of the tissue and the appearance of the speckle pattern, subjectively judged. One exception is the notch at $x=27 \mathrm{~mm}, z=26 \mathrm{~mm}$ in Fig. 7 , which is absent in the compound images based on the angles $0^{\circ}, \pm 7^{\circ}$, and $\pm 14^{\circ}$, but present on the " $21^{\circ}: 7^{\circ}: 21^{\circ}$ " image. This notch is not present in Fig. 6. Repeating the operation of removing the two most extreme angles, so that eventually only the $0^{\circ}$ image is left, will each time result in a slight worsening of the delineation of the tissue and increase in variation due to speckle. Also, artifacts such as those due to reverberations (e.g., Fig. 7, $x=20-27 \mathrm{~mm}, z>31 \mathrm{~mm}$ ) increases in intensity.

Other combinations of angles, as illustrated in the lower row of Figs. 7 and 8, does not appear advantageous. The four angles used to create the image to the left in this row $\left(-21^{\circ},-7^{\circ}, 7^{\circ}\right.$, and $21^{\circ}$ ) probably give the expected improvement in speckle reduction, but the distinct shape and orientation of the psf becomes more dominant. This can especially be seen at $x=27 \mathrm{~mm}$, $z=26 \mathrm{~mm}$ in Fig. 7, where the above-mentioned white notch into the black region seems to be an artifact, as it is not present 
TABLE I

SPECKLe SNR for Adipose and Fibrous Tissues as Well as CONTRAst Between Adipose and Fibrous Tissue Regions, for VARIOUS IMAGING COMBINATION SCHEMES. SAI = Single-ANGLE IMAGE. SNRs Is ONLY CALCULATED FOR "MEAN" AND "SAI"

Method

\begin{tabular}{|c|c|c|c|c|c|c|}
\hline mean & $-21^{\circ}$ & $-14^{\circ}$ & $-7^{\circ}$ & $0^{\circ}$ & $7^{\circ}$ & $14^{\circ}$ \\
\hline mean & & $-14^{\circ}$ & $-7^{\circ}$ & $0^{\circ}$ & $7^{\circ}$ & $14^{\circ}$ \\
\hline mean & & & $-7^{\circ}$ & $0^{\circ}$ & $7^{\circ}$ & \\
\hline mean & $-21^{\circ}$ & & $-7^{\circ}$ & & $7^{\circ}$ & \\
\hline mean & & $-14^{\circ}$ & & $0^{\circ}$ & & $14^{\circ}$ \\
\hline mean & & & $-7^{\circ}$ & & $7^{\circ}$ & \\
\hline mean & & $-14^{\circ}$ & & & & $14^{\circ}$ \\
\hline mean & $-21^{\circ}$ & & & & & \\
\hline SAI & $-21^{\circ}$ & & & & & \\
\hline SAI & & $-14^{\circ}$ & & & & \\
\hline SAI & & & $-7^{\circ}$ & & & \\
\hline SAI & & & & $0^{\circ}$ & & \\
\hline SAI & & & & & $7^{\circ}$ & \\
\hline SAI & & & & & & $14^{\circ}$ \\
\hline SAI & & & & & & \\
\hline $\max$ & $-21^{\circ}$ & $-14^{\circ}$ & $-7^{\circ}$ & $0^{\circ}$ & $7^{\circ}$ & $14^{\circ}$ \\
\hline median & $-21^{\circ}$ & $-14^{\circ}$ & $-7^{\circ}$ & $0^{\circ}$ & $7^{\circ}$ & $14^{\circ}$ \\
\hline rms & $-21^{\circ}$ & $-14^{\circ}$ & $-7^{\circ}$ & $0^{\circ}$ & $7^{\circ}$ & $14^{\circ}$ \\
\hline eomean & $-21^{\circ}$ & $-14^{\circ}$ & $-7^{\circ}$ & $0^{\circ}$ & $7^{\circ}$ & $14^{\circ}$ \\
\hline mem & $-21^{\circ}$ & $-14^{\circ}$ & $-7^{\circ}$ & $0^{\circ}$ & $7^{\circ}$ & $14^{\circ}$ \\
\hline
\end{tabular}

$\Delta \theta$
$7^{\circ}$
$7^{\circ}$
$7^{\circ}$
$14^{\circ}$
$14^{\circ}$
$14^{\circ}$
$28^{\circ}$
$42^{\circ}$
-
-
-
-
-
-
-
$7^{\circ}$
$7^{\circ}$
$7^{\circ}$
$7^{\circ}$
$7^{\circ}$

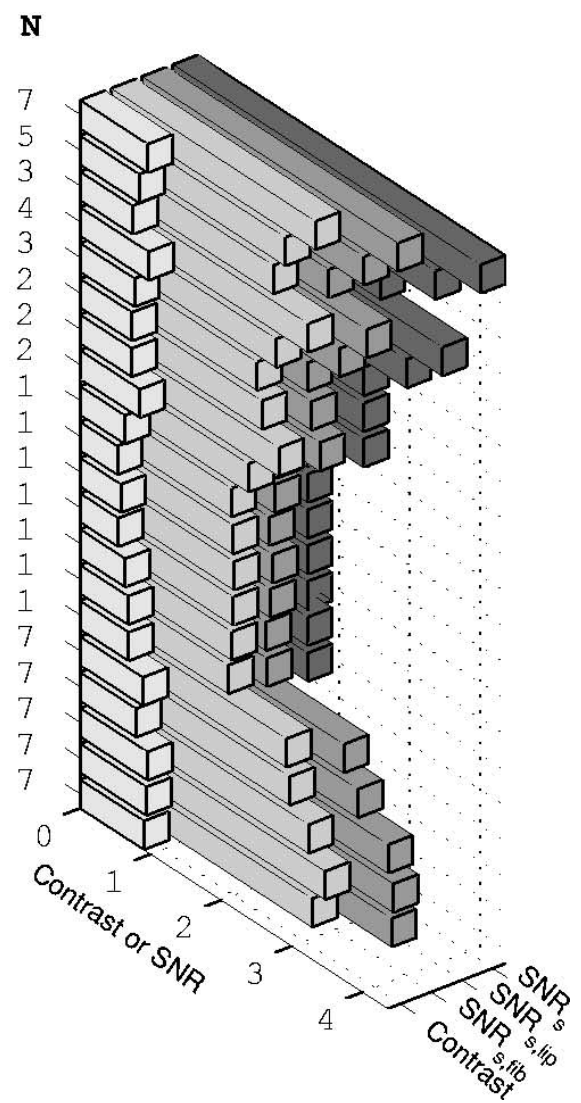

in any of the angles numerically smaller than $21^{\circ}$. This observation is even more accentuated for the angle combination $-21^{\circ}$ and $21^{\circ}$.

Both scan planes contain anisotropic tissue. Scan plane $y_{1}$ contains some muscular tissue with fiber orientation more or less perpendicular to the $0^{\circ}$ beam: This region appears very echogenic on the $0^{\circ}$ and $7^{\circ}$ images in Fig. $4(x=20-24 \mathrm{~mm}$, $z=22-26 \mathrm{~mm})$. Thus, when including more of the remaining SAI in the compound image, the echogenicity of this anisotropic region decreases. As the angle of the preferred direction of anisotropic tissue is unknown in a given clinical scanning situation, it can be argued that compounding results in more correct images. On the other hand, use of the variation of intensity with angle for tissue identification is an obvious aspect to investigate further.

In order to make the images more quantitatively correct for the purpose of tissue classification, the median and mem image formations were introduced to try to minimize the strong contributions from specular reflectors orientated perpendicular to any of the beam angles. Such variations are most pronounced when imaging tubular structures cross-sectionally [8], and less pronounced in the more common tissue geometries in Fig. 6.

The median and mem images in Fig. 9 do not distinguish themselves much from the fully compounded images in Figs. 7 and 8 (upper left image). However, there is one difference: Some of the white short lines (partly shaped as the psf at mainly high beam angles and located perpendicular to the beam direction) have disappeared in the mem image and even more so in the $m e$ dian image. This is be a desirable property, when the goal is to reduce the influence of angle-dependence of anisotropic tissue. Thus, the median and/or the mem operator could play some role in quantitative imaging. Another advantage is the absence of the notch at $y=y_{1}, x=27 \mathrm{~mm}, z=26 \mathrm{~mm}$ in the median and mem images (this notch is not present on the left anatomical photograph in Fig. 6).

A drastic way to reduce the influence of specular reflectors, is to calculate the minimum, instead of the median. However, compared to the mean operator with the same number of angles, the minimum operator results in a $10-\mathrm{dB}$ reduction in the overall levels. Likewise, the speckle SNR and the contrast get reduced with about a third.

The rms approach in Fig. 10 enhances strong echos from a given direction (both due to specular targets, isotropic tissue, and random constructive interference due to speckle). Here, the above-mentioned white lines are more accentuated, relative to the mean image. Further investigations of the possible advantages of the rms image should probably be done with isotropic tissue.

The approach involving logarithmization before averaging, results in the pixel values being multiplied instead of averaged. As for the median image, the tendency to feature "white lines" has been reduced, compared to the mean image, with the same number of angles. But some of the dark regions in the mean image, are more bright in the geometric mean image. The overall pixel level is the lowest for the operators considered. 
The maximum operator provides images (Fig. 12) with a very high pixel level and rather strong delineation of the tissue. The image feature a large number of "white lines" orientated in different directions that are perpendicular to the ultrasound beam. Specifically, the maximum operator "pick up" interfaces and fibers that happens to be perpendicular or near perpendicular to one or more of the ultrasound beam angles used.

For all the images presented here, it should be noted that the changes in appearance might vary when viewed dynamically.

\section{B. Quantitative Interpretation}

The results of the quantitative comparisons, shown in Table I, provides an alternative approach to compare the different compound operators. The mean images used to produce this table were created with a realistic combination of SAIs: when the number of SAIs were reduced, this was done by removing the largest angles first. The reason is that-everything else equal-the larger scan angles feature images with a larger psf, larger influence by grating lobes and lower sensitivity. Based on the visual interpretation of the images in Section III-A, these drawbacks cannot counteract the advantage that the extreme angles (e.g., $-21^{\circ}$ and $21^{\circ}$ ) are less correlated (in a broad sense, including the effect of specular reflectors and anisotropic tissue) than the smaller angles (e.g., $-7^{\circ}$ and $7^{\circ}$ ).

The speckle SNR is between 1.7 and 1.9 for all tissue types for all the SAIs. When combining the SAIs with the mean operator, the $\mathrm{SNR}_{\mathrm{s}}$ increases-in general-with the number of images, $N$, as expected. Likewise, for $N=2$, the $\mathrm{SNR}_{\mathrm{S}}$ increases with $\Delta \theta$. When $N=7$, the highest $\mathrm{SNR}_{\mathrm{s}}$ is obtained for the $r m s$, geomean, and mem operator, while the lowest $\mathrm{SNR}_{\mathrm{S}}$ is obtained for the maximum operator.

For the contrast, the same observations as above can be made for all operators. However, the level of contrast features a relatively high variation for the SAIs, which can be explained by angle-dependence of at least one of the tissues. This also has an effect on the contrast of the compound images.

When comparing the $\mathrm{SNR}_{\mathrm{S}}$ for adipose and fibrous tissues, it is seen that the level is close to 1.91 for adipose tissue but only about 1.7 for fibrous tissue. This is most likely due to the fact that fibrous tissue contain relatively few organized scattering structures and, therefore, does not feature "fully developed speckle." In such a case, the $\mathrm{SNR}_{\mathrm{s}}$ drops below 1.91 [13].

If the $\mathrm{SNR}_{\mathrm{s}}$ for adipose tissue is compared with the theoretical $\mathrm{SNR}_{\mathrm{s}}$, a consistent agreement (within \pm 1 standard deviation) for the SAIs is seen. However, for any combination of images using the mean operator, the experimental value is lower. Three possible reasons for this are:

- While observed from one angle, the adipose tissue appears as tissue with many randomly located scatterers. But the semi-periodic structure of the adipose tissue [as seen in Fig. 3(a)] makes the echo observed from another angle correlated with the first echo. Thus, the structure of the tissue adds an extra contribution to the level of the correlation coefficient between echoes from neighboring angles.

- The adipose regions also contain some thin sheets of fibrous tissue (septa) with weak but relatively large interfaces.
- The adipose tissue regions that are combined in this study are located at different depths and with different tissues between region and transducer. Different depths probably only have a small impact. The attenuation and disturbance of the overlying tissue, might have an effect because attenuation will decrease the bandwidth, thereby making the signals more correlated.

If the theoretical column of results in the Table I is calculated by assuming that the correlation coefficient between two SAIs, separated $7^{\circ}$ and $14^{\circ}$, is $\sigma=0.55$ and $\sigma=0.3$, respectively, then the theoretical and experimental values agree quite well $(\sigma$ values found partly by trial and error). Whether this increase in $\sigma$, relative to the value of $\sigma=0.2$ used in (11), is attributable to anisotropy of the target tissue could be investigated further by computer simulations, with programs such as Field II [6].

\section{Limitations}

Eventually, a few limitations and error sources associated with this study should be mentioned.

The sizes of the adipose and fibrous regions used here were: $78 \pm 48 \lambda^{2}$ and $29 \pm 21 \lambda^{2}$, respectively. These tissue areas-while realistic sizes when dealing with carotid plaque [14] — might be somewhat too small to ensure statistically stable estimates in all circumstances; In another phantom study by us [8], a region of $963 \lambda^{2}$ seemed sufficient. This could be an additional explanation (additional to the angle-dependence), that the maximal contrast of 0.97 was found in line 4 of Table I and not line 1.

Even though great care was taken to slice the tissue at the exact marker locations, some ultrasound scan planes could have been offset up to $0.2 \mathrm{~mm}$ from the plane of the macroscopic image.

The tissue type was mainly identified from the histological image, which represents tissue inside a "plane" with thickness $5 \mu \mathrm{m}$. Because the ultrasound beam is much wider, $1-2 \mathrm{~mm}$, there is a (small) risk, that more tissue types were present, than actually identified from the histological slice.

\section{CONCLUSION}

Compound images from seven different compound operators have been studied visually and quantitatively. For the mean operator, when increasing the number of SAIs, the delineation of the tissue increased when adding SAIs up to $\pm 14^{\circ}$. When adding additional SAIs, the improvement is debatable. The speckle SNR and contrast increased with increasing number of SAIs.

The rms and geomean operators, which in nature are close to the mean operator, did provide some small improvements in $\mathrm{SNR}_{\mathrm{S}}$ and contrast, relative to the mean operator. On the other hand, these images performed worse in the visual analysis. Thus, based on this study, it cannot be recommended to average the logarithmitized images.

In an attempt to obtain more quantitatively correct images, the median and mem operators were introduced to try to reduce the angle-dependence in the image due to anisotropic tissue. These operators provided differently looking (but not necessarily less 
correct) images and good improvements in $\mathrm{SNR}_{s}$ and contrast, especially for mem.

It is finally noted that the improvement in $\mathrm{SNR}_{s}$ was not as large for the kind of structured tissue used in this study, as can be obtained for completely unstructured tissue.

\section{ACKNOWLEDGMENT}

The support by the Danish Technical and Medical Research Councils to CADUS is gratefully acknowledged. The authors gratefully acknowledge the help by: instrument maker $\mathrm{K}$. Martinsen for making the fixtures for the transducer and for handling the biological specimens, T. Brandt for recording the ultrasound and microscopic data of the porcine tissue and J. Hansen for providing the recording software. Finally, the authors appreciate the valuable discussions with Prof. L. K. Hansen and Assoc. Prof. J. Larsen, both of the IMM, Technical University of Denmark.

\section{REFERENCES}

[1] M. Berson, A. Roncin, and L. Pourcelot, "Compound scanning with an electrically steered beam," Ultrason. Imag., vol. 3, pp. 303-308, 1981.

[2] C. B. Burckhardt, "Speckle in ultrasound B-mode scans," IEEE Trans. Sonics Ultraoson., vol. 25, no. SU-1, pp. 1-6, 1978.

[3] D. A. Carpenter, M. J. Dadd, and G. Kossoff, "A multimode real time scanner," Ultrasound Med. Biol., vol. 6, pp. 279-284, 1980.
[4] R. Entrekin, P. Jackson, J. R. Jago, and B. A. Porter, "Real time spatial compound imaging in breast ultrasound: Technology and early clinical experience," Medicamundi, vol. 43, no. 3, pp. 35-43, Sept. 1999.

[5] A. Hernandez, O. Basset, P. Chirossel, and G. Gimenez, "Spatial compounding in ultrasonic imaging using an articulated scan arm," Ultrasound Med. Biol, vol. 22, no. 2, pp. 229-238, 1996.

[6] J. A. Jensen and N. B. Svendsen, "Calculation of pressure fields from arbitrarily shaped, apodized, and excited ultrasound transducers," IEEE Trans. Ultrason., Ferroelect., Freq. Contr., vol. 39, pp. 262-267, Mar 1992.

[7] M. S. Jensen, J. E. Wilhjelm, B. Sahl, T. Brandt, K. Martinsen, S. K. Jespersen, and E. Falk, "A method to obtain reference images for evaluation of ultrasonic tissue characterization techniques," Ultrasonics, vol. 40, no. 1-8, pp. 89-94, 2002.

[8] S. K. Jespersen, J. E. Wilhjelm, and H. Sillesen, "Multi-angle compound imaging," Ultrason. Imag., vol. 20, pp. 81-102, 1998.

[9] D. Kececioglu, Reliability Engineering Handbook. Englewood Cliffs, NJ: Prentice-Hall, 1991, vol. 1, pp. 469-476.

[10] P. M. Shankar and V. L. Newhouse, "Speckle reduction with improved resolution in ultrasound images," IEEE Trans. Sonics Ultraoson., vol. SU-32, no. 4, pp. 537-543, 1985.

[11] D. P. Shattuck and O. T. V. Ramm, "Compound scanning with a phased array," Ultrason. Imag., vol. 4, pp. 93-107, 1982.

[12] G. E. Trahey, S. W. Smith, and O. T. V. Ramm, "Speckle pattern correlation with lateral aperture translation: Experimental results and implications for spatial compounding," IEEE Trans. Ultrason., Ferroelect., Freq. Contr., vol. UFFC-33, no. 3, pp. 257-264, 1986.

[13] T. A. Tuthill, R. H. Sperry, and K. J. Parker, "Deviations from Rayleigh statistics in ultrasonic speckle," Ultrason. Imag., vol. 10, pp. 81-89, 1988.

[14] J. E. Wilhjelm, M.-L. M. Grønholdt, B. Wiebe, S. K. Jespersen, L. K. Hansen, and H. Sillesen, "Quantitative analysis of ultrasound B-mode images of carotid atherosclerotic plaque: Correlation with visual classification and histological examination," IEEE Trans. Med. Imag., vol. 17, pp. 910-922, Dec. 1998. 